\title{
Mathematical Modelling of Tsunami Propagation
}

\author{
${ }^{1}$ EZE, C. L.; ${ }^{2}$ UKO, D. E.; ${ }^{3}$ GOBO, A. E.; ${ }^{4}$ SIGALO, F. B.; ${ }^{5}$ ISRAEL-COOKEY, C. \\ ${ }^{1 \&} 3$ Institute of Geosciences and Space Technology, Rivers State University of Science and Technology, Port Harcourt, Nigeria. \\ ${ }^{2 \&}{ }^{4}$ Department of Physics, Rivers State University of Science and Technology, Port Harcourt, Nigeria. \\ ${ }^{5}$ Department of Mathematics and Computer, Rivers State University of Science and Technology, Port Harcourt, Nigeria. \\ safeearthconsult@yahoo.com.auPhone:08033108660
}

\begin{abstract}
The generation of tsunamis with the help of a simple dislocation model of an earthquake and their propagation in the basin are discussed. In this study, we examined the formation of a tsunami wave from an initial sea surface displacement similar to those obtained from earthquakes that have generated tsunami waves and its propagation through the sea to the shore. Linear shallow water wave equations were employed to explain the propagation of the waves in the open sea while nonlinear wave equations were introduced to explain the behaviour of the wave near the shore. The influence of the Coriolis force on the propagation of tsunami was shown to become very important when the tsunami travel distance is significant in relations to the earth's complete rotation time. The group velocity of tsunami waves which is the velocity of wave energy propagation and its independence on the wave number was demonstrated. @ JASEM
\end{abstract}

The term shallow water wave describes a wave which has a wavelength much greater than the depth of the water it is propagating through. Tsunamis, which have very long wavelengths (10's to 100's km) always travel as shallow water waves even in deep oceans. Although there are other causes of tsunamis, about $90 \%$ of tsunamis of the world are bound up with underwater earthquakes (Jaiwal et al., 2008). Three stages of tsunami development are usually distinguished: (a) formation of a localized initial disturbance and its evolution near the source; (b) propagation of waves in the open ocean; (c) propagation of waves in shallow water and on the shore. The development of numerical models to describe tsunami wave generation, propagation and interaction with complicated topography such as bays or harbours and the resulting flooding has advanced to the stage where they are useful tools for determining the tsunami hazard in local regions. The most common model used to describe tsunami generation, propagation and flooding is the linear, shallow water, long wave model that neglects both the shoaling effect of the shore and the Coriolis effect during a long tsunami travel. Nonlinear wave equations are very important at the shore where most of the damages by tsunami are experienced while Coriolis effect must be considered for teletsunami.

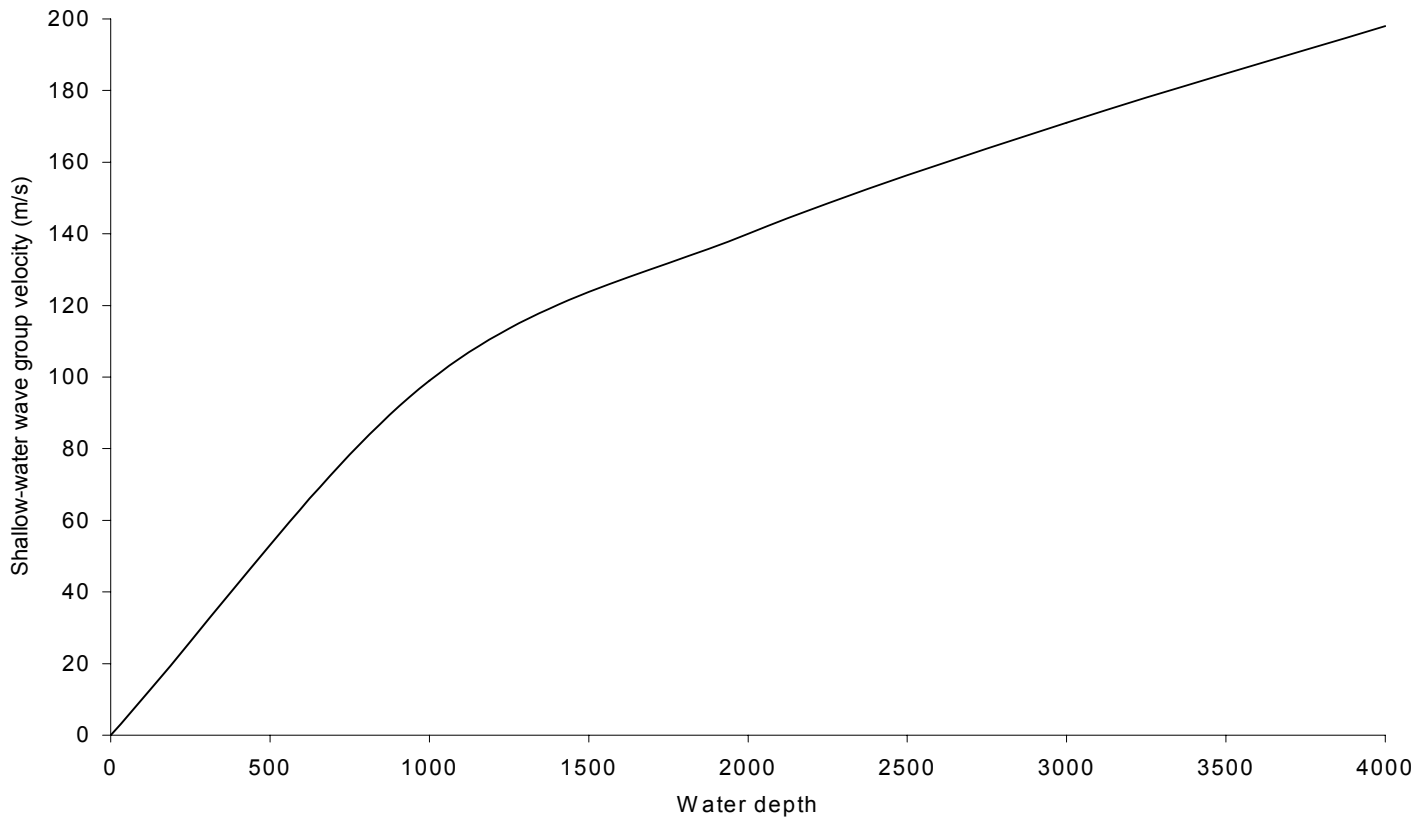

Figure 1.: Tsunami velocity as a function of water depth 


\section{Tsunami wave propagations}

The shallow water wave equations describe a number of physical features including wave dynamics where disturbances in the sea surface height are moving as waves. The basic linear shallow water wave equation of' tsunami generation by small bottom deformations in homogeneous ocean of constant depth $\mathrm{H}$ neglecting the stresses at the surface and bottom, the Coriolis force, and the viscous terms are given as:

$$
\begin{aligned}
& \frac{\delta U}{\delta t}+g H \frac{\delta \eta}{\delta x}=0 \\
& \frac{\delta V}{\delta t}+g H \frac{\delta \eta}{\delta y}=0 \\
& \frac{\delta \eta}{\delta t}+\left(\frac{\delta U}{\delta x}+\frac{\delta V}{\delta y}\right)=0
\end{aligned}
$$

$\eta$ is the vertical displacement of the water surface above the $\mathrm{e}$ quipotential surface, $\mathrm{t}$ is elapse time, $\mathrm{U}$ and $\mathrm{V}$ are the horizontal and vertical components of the water surface, $\mathrm{x}$ and $\mathrm{y}$ are the spatial coordinates of the wave and $g$ is the gravity acceleration (Li and Raichlen, 2001., Zahibo, et al., 2003).

Combining these equations it can be shown that

$$
\frac{\partial^{2} \eta}{\partial t^{2}}-g H \nabla^{2} \eta=0
$$

The corresponding equation in one-dimension derived from equation (4) is:

$$
\frac{\partial^{2} \eta}{\partial t^{2}}-g H \frac{\partial^{2} \eta}{\partial x^{2}}=0
$$

This equation has a wave form and we thus introduce a wave solution in the form $\eta \approx e^{i(k x-\varpi t)}$. Inserting this expression into equation (5) we find that $\eta \approx e^{i(k x-\varpi t)}$ is a solution if and only if :

$\omega=\sqrt{g H} k$

where $\omega$ is the wave frequency in radian and $k$ is the wave number.

Equation (6) is the dispersion relation and characterizes how the frequency must be related to the wave number in order to fulfill equation (5).

Using ordinary theory for surface waves, the dispersion relation is therefore:

$$
\omega^{2}=g k \tanh (k H)
$$

This shows that the tsunami waves must be considerably longer than the depth of the ocean for the results of their treatment as shallow waves to be valid. The average depth of the Pacific Ocean is shallow waves in the ocean valid (Fuji et al., 2001).

\section{Tsunami waves at the shore}

Shallow water approximation is valid if the water depth is much less than the wavelength $(\lambda)$. In this case, $H<<\lambda, k H<<1, \quad$ and $\tanh (k H)=k H$ and nonlinear effects can be neglected.

As the long wave with small amplitude enters shallow coastal waters, the solution contradicts the assumptions of the shallow water wave equation. Tsunami wave propagating over the continental shelf towards the shoreline is transformed mainly by shoaling, refraction and reflection. In this region, nonlinearities cannot be neglected anymore and the full nonlinear shallow water equations must be applied to solve the problem (Casulli and Walters 2000). Guyenne and Grilli (2002) have set the limit of shallow-water and deep-water dispersion relations at $H<\lambda / 11$ and $H>\lambda / 4$ respectively. Detailed bathymetric information and assumed wave length at the shore are required to set these boundaries in the ocean. Tsunami evolution is very important near shore where the waves are dangerous and destructive. The assumptions underlying the tsunami wave equations at the open sea fail near the shore and the bottom topography must be considered. To obtain a solution we assume a simple form for the bottom topography such that

$$
h(x)=-a x
$$

where $h(x)$ is the variable depth of the basin, $\mathrm{x}$ axis

is directed to the shoreline and $\mathrm{s}$ is a constant. Under this condition the tsunami wave takes the form of nonlinear wave equation:

$$
\begin{aligned}
& \frac{\partial \eta}{\partial t}+u \frac{\partial u}{\partial x}+g \frac{\partial \eta}{\partial x}=0 \\
& \frac{\partial \eta}{\partial t}+\frac{\partial}{\partial x}[(h+\eta) u]=0
\end{aligned}
$$

\section{Tsunami equations with Coriolis}

The equations that neglect the Coriolis acceleration can be regarded as valid only as long as the tsunami travel distance remains insignificant in relations to the earth's complete rotation time. When a tsunami propagates over long distances, the Coriolis acceleration terms must be introduced to account for the fact that the earth, frame of reference with respect to which the wave is propagating, is rotating.

The rotation of the earth can strongly affect the tsunami characteristics near the region of formation of tsunamis. Owing to the rotation of the earth, each moving particle of the water is under the influence of 
a Coriolis force. Therefore, tsunami generation is generally accompanied by the formation of internal waves and vortical motions (Johnson, 2003).

During this process some part of the energy, which is transmitted to the ocean with the seismic bottom motions, accumulates in the region of the disturbance. This leads to a reduction of the barotropic wave energy and tsunami amplitude. The direction of the tsunami radiation varies and the energy flow transferred by the waves is redistributed (Pelinovsky et al., 2001)

The integrated equations for linear long waves with Coriollis force in the spherical coordinate system (longitude $\varphi$ and latidute $\theta$ ) can be shown to be

$$
\begin{aligned}
& \frac{\partial Q_{\varphi}}{\partial t}=\frac{g H}{R \sin \theta} \frac{\partial \eta}{\partial \varphi}-f Q_{\theta} \\
& \frac{\partial Q_{\theta}}{\partial t}=\frac{g H}{R} \frac{\partial \eta}{\partial \theta}-f Q_{\theta} \\
& f=2 \Omega \cos \theta
\end{aligned}
$$

where $\mathrm{R}$ is the radius of the earth, $Q$ is the flow rate and $\Omega$ is the rotation vector of the earth

\section{Phase and group velocities}

It is important to consider the group velocity of tsunami waves since it is the velocity of wave energy propagation. For the non-dispersive waves the phase velocity $c_{o}$ is given as

$$
c_{o}^{2}=(g \lambda / 2 \pi) \tanh (2 \pi H / \lambda)
$$

. Since $H<<\lambda$

$c_{o}=\sqrt{g H}$

Group velocity by definition $c_{g}$ is:

$$
c_{g}=\frac{\partial \omega}{\partial k}
$$

By using the approximation for the dispersion relation, we obtain:

$c_{g}=\sqrt{g H}=c_{o}$

The group velocity which here is equal to the phase velocity, is independent of the wave number $k$; so we find that tsunami waves are non-dispersive. Accordingly, we see that all waves travel at the same speed irrespective of the wave number or frequency. This implies that any given form of an initial disturbance in the sea surface will preserve its form.

One notable feature of tsunami waves is therefore, that their speed depends only on the depth of the ocean. Thus when reaching shallow water the waves reach an area with slower propagation speed. The waves will tend to bend towards areas with shallow water since according to Snell's law; waves tend to bend towards areas with lower propagation speed. Thus, when long ocean waves enter a coastline the wave propagation will change direction such that the waves more or less will come in perpendicular to the coastline. For this reason capes usually receive more wave power that bays.

The group velocity variation computed from the average depth $(4000 \mathrm{~m})$ of the Pacific Ocean to the shore as the water depth approaches zero is shown in Figure 1 . The group velocity could be as much as 200 $\mathrm{m} / \mathrm{s}$ which is about the speed of sound in air. As the wave speed decreases, the wave amplitude increases. The amplitude of shallow water waves is approximately:

$$
\frac{A_{s}}{A_{d}}=\sqrt{\frac{C_{d}}{C_{s}}}
$$

where $\mathrm{A}_{\mathrm{s}}$ and $\mathrm{C}_{\mathrm{s}}$ are shallow water amplitude and velocity, and $A_{d}$ and $C_{d}$ are deep water amplitude and velocity. This approximation fails for breaking waves otherwise the amplitude would increase to infinity as water depth approaches zero. In order to keep their frequency constant, their wavelength decreases. The energy of the wave is transmitted as kinetic energy and potential energy. The kinetic energy is tied up in the motion of the water while the potential energy is tied up in gravitational potential that is the amplitude of the wave. However, when the wave approaches the shore and enters shallow water, the kinetic energy of the water near the bottom is converted into potential energy of the water at the top and this actually explains why the height of the tsunami wave increases as the wave approaches the shore.

CONCLUSIONS: Accurate prediction of tsunami hazards demands that the tsunami propagation be treated in two separate phases: linear shallow water waves in the open sea and as nonlinear wave near the shore. Bathymetric data are required to set the boundary between the constant depth of the open sea and the variable depth near the shore. Whereas Coriolis effects may not be considered as important in local tsunamis, these effects must be integrated into the treatment of teletsunamis to ensure a more accurate determination of their point of arrival.

\section{REFERENCES}

Casulli, V. and Walters, R.A. (2000) An unstructured grid, three-dimensional model based on the shallow water equations; International Journal for Numerical Methods in Fluids; Vol. 32; No. 3; 331-348 
Fujima, K., Masamura, K., and Goto, C. (2002).Theoretical examinations of long-wave damping by sea bottom friction. Coastal Engineering journal, 44 (3): 217-245

Guyenne, P. and Grilli ,S. T (2003). "Computations of three-dimesional overturning waves in shallow water: Dynamics and Kinetics." Proc. $13^{\text {th }}$ Int. Offshore and Polar Eng. Conference.,Honolulu ,Hawaii ,USA,.International Society of the Offshore and Polar Engineers.

Jaiswal, R. K., Rastoqi, B. K. and Murty, T. S. (2008). Tsunamigenic Sources in The Indian Ocean. Science of Tsunami Hazards, Vol. 27, No. 2, 32-48

Johnson ,R.S. (2003).“The Classical Problem of Water Waves :A Reservoir of Intergrable and
Nearly-

IntergrableEqations."Journal.Nonl.Math.Phys.10 Suppl.1,72-92.

Li, Y. and Raichlen, F. (2001) Solitary wave runup on plane slopes. Journal of Waterway, Port, Coastal, and Ocean Engineering. Vol. 127. No. 1. 33-44

Pelinovsky ,E.; Talipova, T. ;Kurkin, A.; and Kharif, C (2001).'Nonlinear Mechanism of Tsunami Wave Generation By Atmospheric Disturbances." Natural Hazard And Earth Science.1,243-250.

Zahinbo, N.;Pelinovsky, E.;Yalciner, A.C. ;Kurkin, A.;Koselkov ,A.;and Zaitsev, A (2003). "The 1867 Virgin Island Tsunami." Natural Hazard and Earth Sci.3,367-376. 